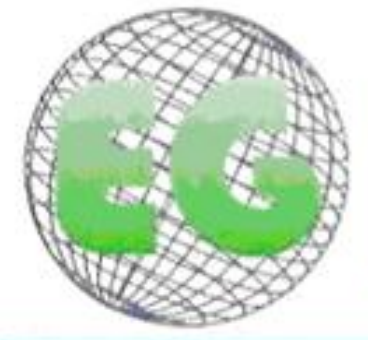

ISSN 1695-6141

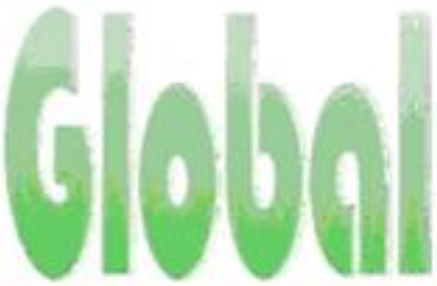

Revista electrónica trimestral de Enfermería

www.um.es/egloball

Julio 2016

\title{
REVISIONES
}

\section{El diseño de preguntas clínicas en la práctica basada en la evidencia. Modelos de formulación}

Design of clinical questions in evidence-based practice. Formulation models

\section{*Martínez Díaz, Juan Daniel **Ortega Chacón, Verónica *Muñoz Ronda, Francisco José}

\author{
*Enfermero. Hospital Torrecárdenas. Profesor Asociado. Departamento de Enfermería, Fisioterapia y \\ Medicina. Universidad de Almería. E-mail: ¿dani7@terra.com **Enfermera. Hospital Torrecárdenas.
} Almería. España.

Palabras clave: pregunta; formulación; práctica basada en la evidencia; estructura

Keywords: evidence based practice; question; formulation; framework.

\section{RESUMEN}

Objetivo: Identificar estructuras estandarizadas para la formulación de preguntas clínicas en el marco de la práctica basada en la evidencia.

Método: Se realizó una revisión de la literatura. La búsqueda bibliográfica se efectuó en las bases de datos MEDLINE, CINAHL, EMBASE y LILACS, y en el buscador académico Google Scholar. Se efectuaron estrategias de búsqueda sensibles y acordes a cada base de datos. Límites de búsqueda: intervalo de tiempo (enero de 1995 a abril de 2015), idioma (artículos en inglés). Se usaron palabras clave libres y descriptores del Medical Subject Headings (MeSH) pertinentes: evidence based practice, question, formulation, well-built question, framework.

Resultados: Se encontraron 10 manuscritos que aportaron el diseño original de estructuras para la formulación de preguntas clínicas en el ámbito de la práctica basada en la evidencia. El modelo PICO es la estructura más conocida y comúnmente utilizada en investigación cuantitativa y de él derivan los modelos PICOT, PICOTT, PICOS, PIPOH, PECORD, PESICO. En el campo de la gestión sanitaria la estructura ECLIPSE se erige para formulación de preguntas relacionadas con la gestión. Por último, para la búsqueda de evidencias cualitativas se han configurado los modelos SPICE y SPIDER adecuando sus componentes al fenómeno cualitativo.

Conclusiones: Estos modelos estandarizadas se comportan como un instrumento idóneo para guiar la estrategia de búsqueda y delimitar el área de interés. Dada la gran variedad de piezas que integran las estructuras, su conocimiento exhaustivo acrecienta sus usos potenciales. Estas estructuras no deben ser consideradas como una guía rígida a la que ceñirse ineludiblemente. 


\section{ABSTRACT}

Objective: To identify standard patterns for the construction of clinical queries in the context of evidence-based practice scenario.

Method: A literature review was carried out. A sensitive and specific search was driven into MEDLINE, CINAHL, EMBASE and LILACS databases, along with academic search engine Google Scholar. Search limits: publishing period (January 1995 to April 2015) and language (articles in English). Free key words and descriptors of Medical Subject Headings (MeSH) used were: evidence based practice, question, formulation, well-built question, framework.

Results: 10 manuscripts providing the original design of patterns for the formulation of clinical questions in the field of evidence-based practice were found. The PICO model is the best and broadly known, and it is commonly used in quantitative research. PICO model has also been the foundation to PICOT, PICOTT, PICOS, PIPOH, PECORD, and PESICO models. In the field of health management, ECLIPSE model highlights for management-related queries. Finally, SPIDER and SPICE models have been commonly used for qualitative research questions, due to their advantage for adapting its components to the qualitative phenomenon.

Conclusions: These standard models behave as suitable tools to guide search strategies and define fields of interest. Given the variety of elements that configure these patterns, their comprehensive knowledge increases its potential applications. These structures should not be considered as a rigid rule. Moreover they draw a wide and flexible scope to drive effective search strategies.

\section{INTRODUCCIÓN}

Desde mediados de la década de los 90, la práctica basada en la evidencia (PBE) ha experimentado de forma progresiva un papel relevante en la esfera de la atención sanitaria, proporcionando a los proveedores de atención de la salud un marco para la toma de decisiones. La transferencia de la evidencia en la práctica clínica constituye un proceso dinámico que sigue una progresión lógica a través de un sistema multietapas. La mayoría de autores consideran que la elaboración de la pregunta clínica es el primer paso, y el eje a partir del cual se articula todo el proceso ${ }^{1-4}$.

La PBE posibilita transformar una necesidad de información, proveniente de un problema o área de incertidumbre, en una pregunta susceptible de ser contestada. Este cometido constituye uno de los principales desafíos para quienes cultivan la evidencia. La literatura en ciencias de la salud revela que numerosas preguntas quedan sin respuesta debido a las dificultades en su formulación ${ }^{5}$. Una pregunta mal elaborada reduce la probabilidad de recuperar la información pertinente, y genera un elevado e infructuoso consumo de tiempo en la búsqueda.

La formulación de buenas preguntas clínicas es una habilidad básica y un rasgo determinante de la competencia enfermera. Plantearse preguntas es una de las premisas clave del pensamiento crítico, ese pensamiento reflexivo encaminado a la resolución de problemas, que aspira a formular juicios basándose en evidencias ${ }^{6}$.

El escenario clínico cotidiano es el mayor generador de preguntas, pero no es el único. Como indica Icart $^{7}$ el uso de la evidencia no se circunscribe exclusivamente al entorno clínico, sino que se extiende a los ámbitos de la gestión y la docencia. Así, la determinación de ratio/enfermera paciente en una unidad de hospitalización, la asistencia a eventos formativos o una simple demanda de información de un alumno ejemplifican situaciones que engendran preguntas acerca de cuál es la mejor decisión a tomar. 
La demanda de respuestas exhaustivas y precisas implica la elaboración de preguntas bien confeccionadas. Para ello, se dispone de estructuras estandarizadas que organizan la formulación de la pregunta, centrándose en los elementos clave y facilitando a su vez una buena estrategia de búsqueda.

El objetivo de esta revisión es identificar estructuras estandarizadas para la formulación de preguntas clínicas en el contexto de la práctica basada en la evidencia.

\section{MÉTODO}

Se realizó una revisión bibliográfica entre los meses de febrero y abril de 2015 . Fuentes de información: se efectuó una búsqueda de la literatura en las bases de datos bibliográficas MEDLINE, CINAHL, EMBASE, LILACS y CUIDEN PLUS, en el buscador académico Google Scholar y se revisaron manualmente las referencias de los artículos recuperados, con el fin de detectar literatura no encontrada en la búsqueda preliminar. Límites de búsqueda: el periodo de búsqueda se limitó entre enero de 1995 y marzo de 2015. Respecto al idioma se seleccionaron artículos publicados en inglés y español. Se incluyeron todo tipo de estudios. Estrategias de búsqueda: se desplegaron estrategias de búsqueda sensibles y acordes con cada base de datos. Las búsquedas fueron efectuadas usando palabras clave libres y descriptores del Medical Subject Headings (MeSH) pertinentes en inglés, y descriptores en Ciencias de la Salud (DeCS) de BIREME en lengua castellana. Se emplearon sinónimos para refinar la búsqueda. Los términos utilizados en inglés y español fueron: evidence-based practice, question, formulation, well-built question, framework. Se seleccionaron aquellos artículos en los que los términos aparecían en el titulo o el resumen. Los operadores booleanos empleados para formular la cadena de búsqueda fueron: AND y OR. Se usó truncamiento y comillas.

\section{RESULTADOS}

Al aplicar los límites de búsqueda y los criterios de selección se han hallado diez manuscritos que revelan el diseño original de estructuras empleadas para la formulación de preguntas en el contexto de la PBE. Estos modelos se describen a partir de la literatura original.

\section{Estructuras para la formulación de preguntas: el modelo PICO}

En 1995, en la editorial de la revista ACP Journal club, Richardson et al $^{1}$ señalan que toda pregunta bien elaborada viene respaldada por dos premisas. En primer lugar, la pregunta debe ser relevante al problema identificado, es decir, estar centrada en la cuestión que realmente interesa. A continuación, debe articularse de modo que facilite la búsqueda de una respuesta precisa y exhaustiva.

Para alcanzar estos objetivos, los autores proponen una estructura especial integrada por cuatro elementos bien diferenciados, a la que denominan con el acrónimo PICO. Este código nemotécnico facilita recordar los componentes de la estructura: $(P)$ paciente o problema de interés: características del paciente o grupo de pacientes; características del problema o condición, (I) Intervención: intervención principal a considerar (terapéutica, preventiva, diagnóstica, exposición de riesgo, etc.), (C): intervención de comparación: alternativa con la que comparar la intervención principal (hay que tener en cuenta, que en ocasiones no se dispone de una intervención con la 
que comparar) y $(\mathrm{O})$ (outcomes) resultado a valorar: efectos de la intervención, en términos de mejora, efectos secundarios, etc.

En la actualidad, el formato PICO es el más empleado en la construcción de preguntas de indagación, no sólo en el ámbito de la medicina basada en la evidencia, sino en otras disciplinas de ciencias de la salud y en disciplinas no afines ${ }^{8}$.

\section{Modelos derivados de PICO}

A partir de la presentación de PICO, varias han sido las estructuras de formulación de preguntas que se han confeccionado tomando como base su modelo (tabla 1). En algunos casos, estos modelos alternativos han conservado los cuatro elementos primigenios, más la incorporación de algún factor nuevo, mientras que en otros, ciertas variantes han emergido a partir de la modificación de una o alguna de las piezas originales.

Fineout-Overholt y Johnson ${ }^{9}$, en el contexto de la enseñanza de la PBE en enfermería, consideran que la reflexión sobre una situación clínica se establece como el paso previo a la formulación de la pregunta. Para facilitar la elaboración de preguntas, las autoras plantean el requisito de usar un formato estandarizado, e introducen para ello el marco PICOT. Esta estructura de cinco elementos adopta el formato PICO e incorpora la T (time frame) que representa el intervalo de tiempo para que la intervención logre el resultado. Aunque este elemento no siempre se muestra, su inclusión en un formato estandarizado reduce la probabilidad de que el tiempo se pueda omitir en aquellas preguntas en las que es preciso.

Una expansión de PICO muy parecida a la anterior es PICOTT. Desarrollado en 2007 por Schardt et $\mathrm{al}^{5}$, el acrónimo incorpora una doble T. La primera T (type of question) añade información sobre el tipo de pregunta que se realiza (diagnóstico, pronóstico, intervención, etc.) y la segunda (type of study design) hace referencia al mejor diseño de investigación para responder a esa pregunta específica. El uso de este marco ayuda a los profesionales a articular las partes cardinales de la pregunta clínica y facilita el proceso de búsqueda.

Especialmente diseñado para la elaboración de Revisiones Sistemáticas (RS), el Centre for Reviews and Dissemination (CRD) ${ }^{10}$ de la Universidad de York incorpora la estructura PICOS. Desde 1996, el CRD publica su Guía para elaborar RS, cuyo objetivo es ofrecer un método para llevar a cabo la síntesis de la evidencia, basada en un amplio conocimiento de la metodología de la RS. El protocolo de revisión introduce como primer eslabón del proceso la formulación de la pregunta de revisión. Las RS deben establecer preguntas claras, cuyas respuestas aporten información significativa que guíe la toma de decisiones. El marco PICOS incluye todos los elementos de PICO y añade la letra S (study design) de diseño de estudio. Los tipos de estudios incluidos en la revisión van a ser determinantes para la fiabilidad de los resultados. Asimismo, la guía indica que las preguntas de revisión no siempre van a especificar el tipo de diseño de estudio.

Al igual que sucede con las RS, otra de las herramientas que ha posibilitado un nexo de unión entre la investigación y el terreno clínico ha sido las guías de práctica clínica (GPC). Desde finales de los 90, adicionalmente al desarrollo de nuevas guías, se ha sugerido un proceso de adaptación de guías ya elaboradas. Esta adaptación supone utilizar una GPC que ha sido elaborada en una organización con unas características 
culturales determinadas o contexto particular, y modificarla para aplicarla en un entorno diferente. Para orientar este proceso, la colaboración ADAPTE ${ }^{11}$ ha definido un marco metodológico, cuya segunda fase, denominada de adaptación, incluye la identificación de las preguntas clave que la guía intenta responder. Con el objetivo de definir la pregunta, la guía presenta una estructura de cinco elementos, a la que denomina PIPOH. En este marco, la segunda $\mathrm{P}$ se refiere a los profesionales a los que se destina la guía y la $\mathrm{H}$ (health care setting) al contexto en el que se implementará. El resto de elementos se ajustan a los del formato PICO. Aunque $\mathrm{PIPOH}$ fue desarrollado específicamente para el proceso de adaptación de GPC oncológicas, actualmente se está confeccionando un modelo genérico.

Como ya se ha señalado anteriormente, es básico formular preguntas que nos posibiliten la posterior confección de una búsqueda bibliográfica lo más eficiente posible. En este sentido, Dawes et al $^{12}$ desarrollan el modelo de pregunta PECORD con el objetivo de explorar la viabilidad de la identificación de los elementos de esa estructura en los resúmenes de los artículos de revistas médicas y así facilitar la recuperación de información. Partiendo de la estructura PICO, reemplazan el término intervención por exposición (E) lo que posibilita la inclusión de diferentes tipos de estudio (cohortes y casos-controles), e introducen dos nuevos elementos: (D) indica la duración del tratamiento y/o duración hasta que el resultado se evalúa y $(R)$ referida a resultados.

Para concluir con la familia de modelos derivados de $\mathrm{PICO}$, en el ámbito de la patología del habla y del lenguaje, específicamente en la comunicación aumentativa y alternativa, encontramos otra de sus variaciones. Schlosser et $\mathrm{al}^{13}$ plantean PESICO como una estructura más apropiada para ese campo. Esta estructura anexa a PICO los elementos $\mathrm{E}$ y $\mathrm{S}$, donde $\mathrm{E}$ (environments) hace referencia al entorno en el que acontece el problema, y $S$ (skateholders) representa a aquellas personas que tienen un interés en el resultado.

Tabla 1. Componentes del modelo PICO y sus variantes

\begin{tabular}{|c|c|c|c|c|c|c|c|c|c|c|c|c|c|c|}
\hline & 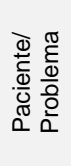 & 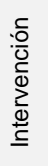 & 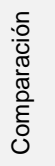 & 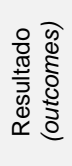 & 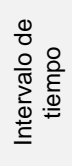 & 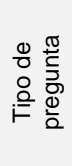 & 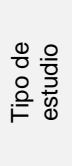 & 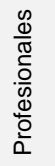 & 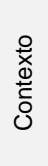 & $\begin{array}{l}: \frac{0}{0} \\
\frac{0}{0} \\
0 \\
\frac{0}{x} \\
ய\end{array}$ & 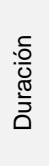 & 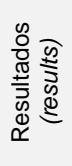 & 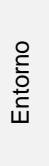 & 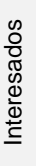 \\
\hline PICO & $a$ & $a$ & $a$ & $a$ & & & & & & & & & & \\
\hline PICOT & $a$ & $a$ & $a$ & $a$ & $a$ & & & & & & & & & \\
\hline PICOTT & $a$ & $a$ & $a$ & $a$ & & $a$ & $a$ & & & & & & & \\
\hline PICOS & $a$ & $a$ & $a$ & $a$ & & & $a$ & & & & & & & \\
\hline PIPOH & $a$ & $a$ & & $a$ & & & & $a$ & $a$ & & & & & \\
\hline PECORD & $a$ & & $a$ & $a$ & & & & & & $a$ & $a$ & $a$ & & \\
\hline PESICO & $a$ & $a$ & $a$ & $a$ & & & & & & & & & $a$ & $a$ \\
\hline
\end{tabular}




\section{Los marcos ECLIPSE, SPICE y SPIDER}

A pesar de que el modelo PICO es el más comúnmente utilizado por los profesionales de la salud para la elaboración de preguntas clínicas, su diseño no se adecúa a toda la gama de preguntas que surgen en el contexto de las ciencias de la salud, circunstancia que ha suscitado la construcción de otros marcos alternativos.

En el campo de la gestión sanitaria, Wildridge y Bell ${ }^{14}$ desarrollan la estructura ECLIPSE dirigida específicamente a la formulación de preguntas relacionadas con la gestión y las políticas de salud. ECLIPSE se cimienta a partir de la evolución de una estructura previa denominada CLIP (grupo de clientes, ubicación, impacto y profesionales). De CLIP conserva los términos (C) grupo de clientes: ¿a quién está destinado el servicio?, (L) ubicación: ¿cuál es la ubicación física del servicio? y $(\mathrm{P})$ profesionales: ¿quién proporciona el servicio?. Asimismo, modifica la (I) que cambia a impacto: ¿cuál es el impacto del servicio?, ¿qué supone el éxito? y ¿cómo se mide? y se complementa con la (E) expectativa: ¿para que requiere el solicitante la información? y la (SE): servicio: para que servicio se busca información: cuidados intermedios, consultas externas, rehabilitación, etc.

Si bien, desde su creación PICO se ha postulado como la mejor herramienta para la formulación de preguntas en metodología cuantitativa, su anatomía no se adecúa a la búsqueda de evidencias cualitativas. El hecho de que sus dos elementos más utilizados, paciente e intervención, estén diseñados para recuperar información eminentemente cuantitativa, junto a la presencia de un comparador, elemento no distintivo de una pregunta cualitativa, respalda esta condición. Con el propósito de cubrir este vacío, se han planteado algunos modelos para su uso en investigación cualitativa.

El modelo SPICE ideado por Booth ${ }^{15}$ en 2004, se introduce en el contexto de la biblioteconomía basada en la evidencia, y es a la postre adoptado por el Instituto Joanna Briggs ${ }^{16}$ como el marco adecuado para guiar la elaboración de las preguntas de revisión en las RS cualitativas. SPICE incorpora los siguientes elementos: (S) escenario: ¿dónde? (el contexto de un estudio), (P) perspectiva: ¿para quién? (la perspectiva descrita por diferentes valores y actitudes), (I) intervención: ¿qué? (el fenómeno de estudio), (C) comparación: ¿comparada con qué? y (E) evaluación: ¿con qué resultado? (la evaluación del proceso y resultados). Los revisores apuntan que no todos los elementos de la pregunta se aplican necesariamente en cada revisión. El uso de este marco se debe considerar más como una guía que como una norma.

Tras diversas propuestas para adaptar el marco PICO al espacio de la metodología cualitativa o bien adoptar estructuras provenientes de otras disciplinas, como ocurre el caso de SPICE, la disponibilidad de un modelo específico para la síntesis de evidencia cualitativa ha venido de la mano de SPIDER.

Cooke et $\mathrm{a}^{17}$ configuran el esquema SPIDER teniendo en cuenta las consideraciones de diversos investigadores sobre los inconvenientes del uso de PICO en la búsqueda de estudios cualitativos ${ }^{18}$. El diseño del nuevo modelo se desarrolla mediante la adecuación de los elementos de PICO, completándose con la adhesión de nuevos componentes, de la siguiente manera: (S) muestra: en investigación cualitativa se utilizan muestras más pequeñas, donde los resultados no están destinados a ser generalizados a la población en general, $(\mathrm{PI})$ fenómeno de interés: la investigación 
cualitativa explora el cómo y el por qué ciertas experiencias, comportamientos y decisiones, (D) diseño: el marco teórico va a determinar el método de investigación a usar, el diseño del estudio va a influir en la robustez y análisis del estudio, (E) evaluación: la evaluación de los resultados puede incluir resultados más subjetivos (opiniones, actitudes, etc.), (R) tipo de investigación (cualitativa, cuantitativa y métodos mixtos): la suma de este elemento tiene la ventaja añadida de que SPIDER puede ser utilizado para estrategias de búsqueda de estudios cuantitativos y de aquellos con métodos mixtos.

\section{CONCLUSIÓN}

La formulación de la pregunta clínica bien construida se erige como una etapa esencial para la práctica basada en la evidencia y una competencia básica en la formación enfermera. A pesar de que en la actualidad es quimérico afirmar que todas nuestras necesidades de información van a poder ser cubiertas con alguno de los modelos presentados en esta revisión, estas estructuras estandarizadas se comportan como un instrumento idóneo que orienta la estrategia de búsqueda y delimita el área de interés en relación al problema planteado. En lugar de considerar estos modelos como diferentes y particulares, es eficaz explorar los elementos que los configuran y usarlos de manera intercambiable cuando sea necesario ${ }^{8}$. Dada la gran variedad de piezas que integran los modelos, su conocimiento exhaustivo acrecienta sus usos potenciales. No obstante, estas estructuras deben ser consideradas más como una lista de recomendaciones que como una guía rígida a la que ceñirse ineludiblemente.

\section{REFERENCIAS}

1. Richardson WS, Wilson MC, Nishikawa J, Hayward, R. The well-built clinical question: A key to evidence-based decisions. ACP journal club 1995; 123: A12-13.

2. Thomson C, Cullum N, McCaughan D, et al. Nurses, information and clinical decision making the real world potential for evidence-based decision in nursing. Evidencebased nursing 2004; 7: 68-72.

3. Straus SE, Richardson W, Glasziou P, Brian R. Medicina basada en la evidencia. Como practicar y enseñar la MBE. Madrid: Elsevier; 2006.

4. Mantzouskas S. A review of evidence-based practice, nursing research and reflection: levelling the hierarchy. Journal of clinical nursing 2008; 17(2):214-223.

5. Schardt C, Adams MB, Owens T, Keitz S, Fontelo P. Utilization of the PICO framework to improve searching PubMed for clinical questions. BMC Medical Informatics and Decision Making 2007; 7: 16.

6. Morales JM. Investigación enfermera en atención primaria (II): la práctica basada en la evidencia. En: Martínez Riera JR, Del Pino Casado R. Enfermería en Atención Primaria. Madrid: DAE; 2006.

7. Icart MT. Enfermería basada en la evidencia. En: Libro de Ponencias: II Congreso de la Asociación Andaluza Enfermería Comunitaria (ASANEC). Málaga; 2000.

8. Davies KS. Formulating the Evidence Based Pratice Questions: A Review of the Frameworks. Evidence Based Library and Information Practice 2011; 6: 75-80.

9. Fineout-Overholt E, Johnson L.Teaching EBP: Asking searchable, answerable clinical questions. Worldviews on Evidence-Based Nursing 2005; 2(3): 157-60.

10. Centre for Reviews and Dissemination. University of York. Systematic Reviews: CRD Guidance for undertaking reviews in health care, 2009. [acceso 3 Mar 2014 ]. Disponible en: http://www.york.ac.uk/inst/crd/pdf/Systematic Reviews.pdf 
11. The ADAPTE Collaboration. The ADAPTE Process: Resource Toolkit for Guideline Adaptation. Version 2.0. 2009. [acceso 20 Mar 2014 ]. Disponible en: http://www.gin.net/document-store/working-groups-documents/adaptation/adapte-resource-toolkit guideline-adaptation-2-0.pdf

12. Dawes M, Pluye P, Shea L, Grad R, Greenberg A, Nie JY. The identification of clinically important elements within medical journal abstracts: Patient population problem, exposure intervention, comparison, outcome, duration and results (PECODR). Informatics in Primary Care 2007; 15(1): 9-16.

13. Schlosser RW, O'Neil-Pirozzi T. Problem formulation in evidence-based practice and systematic reviews. Contemporary Issues in Communication Sciences and Disorders 2006; 33: 5-10.

14. Wildridge V, Bell L. How CLIP bécame ECLIPSE: a mnemonic to assitst in searching fpr health policy/management information. Health Info Libr J 2002; 19 (2): 113-115.

15. Booth A. Clear and present questions: Formulating questions for evidence based practice. Library Hi Tech 2006; 24(3): 355-68.

16. The Joanna Briggs Institute. Joanna Briggs Institute Reviewers' Manual. Adelaide: 2011.

17. Cooke A, Smith D, Booth A. Beyond PICO: the SPIDER tool for qualitative evidence synthesis. Qual Health Res. 2012 Oct; 22(10):1435-43.

Recibido: 10 de octubre 2015; Aceptado: 29 de noviembre 2015

ISSN 1695-6141

(C) COPYRIGHT Servicio de Publicaciones - Universidad de Murcia 